\title{
A novel SOX10 variant in a Japanese girl with Waardenburg syndrome type 4C and Kallmann syndrome
}

\author{
Junpei Hamada (1) ${ }^{1}$, Fumihiro Ochi', Yuka Sei ${ }^{1}$, Koji Takemoto ${ }^{2}$, Hiroki Hirai ${ }^{3}$, Misa Honda ${ }^{4}$, Hironori Shibata ${ }^{4}$, \\ Tomonobu Hasegawa ${ }^{4}$ and Mariko Eguchi id ${ }^{1}$
}

\begin{abstract}
We report the first case of Waardenburg syndrome type 4C and Kallmann syndrome in the same person. The patient, a Japanese girl, presented with bilateral iris depigmentation, bilateral sensorineural hearing loss, Hirschsprung disease, hypogonadotropic hypogonadism, and anosmia. We identified a novel SOX10 variant, c.124delC, p.Leu42Cysfs*67.
\end{abstract}

Waardenburg syndrome (WS), a rare autosomal dominant disorder, is characterized by sensorineural hearing loss and pigmentation abnormalities of the hair, irises and skin $^{1}$. WS is classified into four subtypes, WS1, WS2, WS3, and WS4. WS4 is defined as a complication of Hirschsprung disease. WS4 is genetically heterogeneous, with three causative genes identified ${ }^{2}$. WS, type $4 \mathrm{C}$ (WS4C, OMIM\#613266) is caused by heterozygous mutation in the SOX10 gene, whereas WS, type 4A (WS4A, OMIM\#277580) and WS, type 4B (WS4B, OMIM\#613265) are caused by mutation of EDNRB and $E D N 3$, respectively. SOX10 belongs to the SOX family, the members of which have a high mobility group (HMG) DNA binding domain. SOX10 plays a major role in the development and migration of neural crest cells, which can differentiate into melanocytes, olfactory ensheathing cells, and enteric ganglia neurons ${ }^{1-5}$.

Kallmann syndrome (KS) is a clinically and genetically heterogeneous disorder defined by hypogonadotropic hypogonadism $(\mathrm{HH})$ and olfactory dysfunction. $\mathrm{KS}$ is occasionally associated with hearing loss, which occurs in approximately $5 \%$ of patients ${ }^{6}$. Several genetic variants

\footnotetext{
Correspondence: Junpei Hamada (hamada.jumpei.yn@ehime-u.ac.jp) 'Department of Pediatrics, Ehime University Graduate School of Medicine, Ehime, Japan

${ }^{2}$ Department of Pediatrics, Ehime Prefectural Niihama Hospital, Ehime, Japan Full list of author information is available at the end of the article
}

have been linked to the etiology of KS with hearing loss, including KAL1, FGFR1, FGF8, IL17RD, and $C H D 7^{7-10}$. Recently, variants in SOX10 have been identified in a few KS patients with hearing loss ${ }^{11}$.

Here, we report for the first time a case with both WS4C and KS due to a novel SOX10 variant. The proband was referred to our outpatient clinic at 14.9 years of age for lack of pubertal development. She was born to nonconsanguineous Japanese parents at 39 weeks and 6 days of gestation. The pregnancy and delivery were uncomplicated. Her birth weight and length were $2890 \mathrm{~g}(-0.6 \mathrm{SD})$ and $47.0 \mathrm{~cm}(-1.3 \mathrm{SD})$, respectively. Shortly after birth, bilateral iris depigmentation was noted (Fig. 1a, b). At $24 \mathrm{~h}$ of life, she exhibited abdominal distention, vomiting, and delayed meconium excretion. The intraoperative findings showed caliber changes. The rectal mucosal biopsy sample was positive for acetylcholinesterase staining, which confirmed the diagnosis of Hirschsprung disease. The newborn hearing screening test produced a 'refer' result, and auditory brainstem response (ABR) revealed congenital bilateral sensorineural hearing loss. Thus, she was clinically diagnosed with WS4. Her mental development was normal. On the first visit at 14.9 years of age, her height was $142.2 \mathrm{~cm}(-2.79 \mathrm{SD})$. She showed no pubic hair or breast development (Tanner stage 1). Endocrinological evaluation showed poor gonadotropin response after gonadotropin-releasing hormone stimulation; basal and 

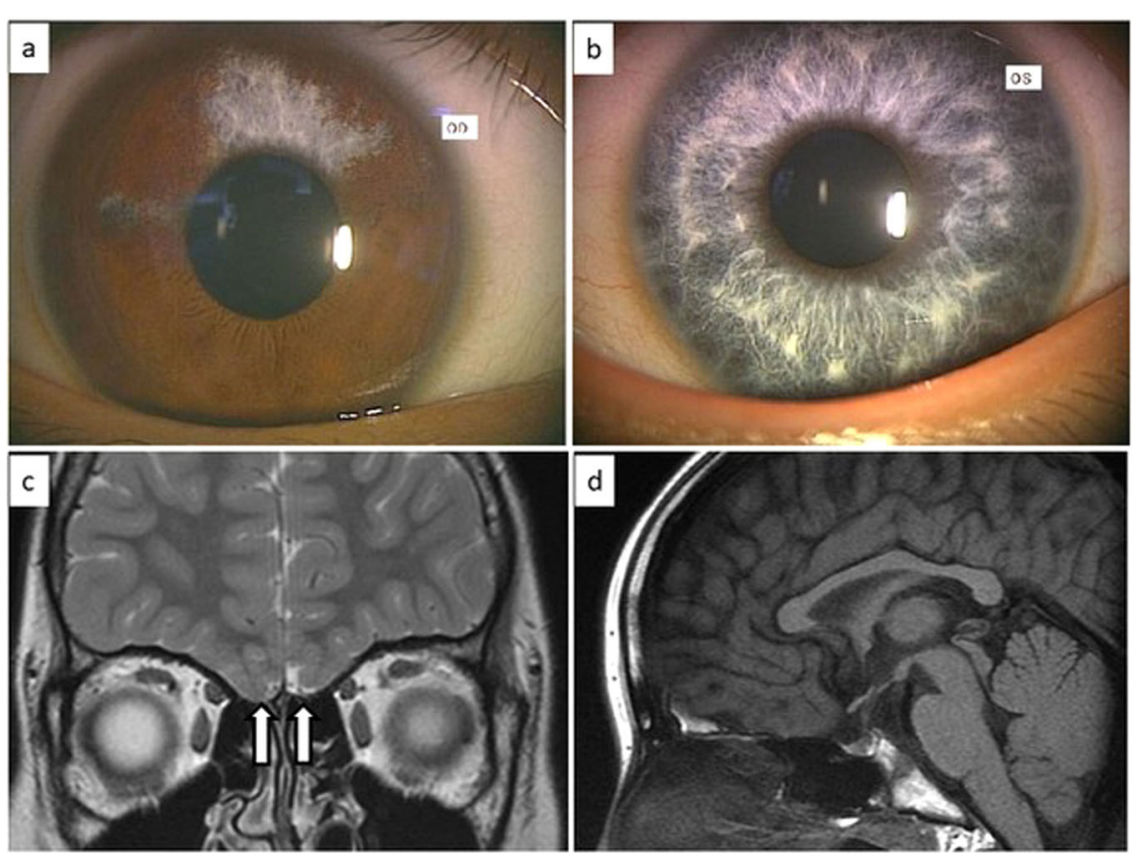

Fig. 1 The picture of iris, and MRI findings. $\mathbf{a}$, b Iris depigmentation. a Right (partial depigmentation). $\mathbf{b}$ Left (complete depigmentation). $\mathbf{c}$, d T2weighted brain magnetic resonance imaging. $\mathbf{c}$ White arrows show olfactory bulb agenesis. $\mathbf{d}$ No abnormalities were noted in the hypothalamus and pituitary.

peak $\mathrm{LH}$ values of $<0.2 \mathrm{mIU} / \mathrm{ml}$ (reference range: $0.4-4.1$ ) and $3.1 \mathrm{mIU} / \mathrm{ml}$ (reference range: $8.5-15.5)$, respectively; basal and peak FSH values of $1.1 \mathrm{mIU} / \mathrm{ml}$ (reference range: 4.8-10.4) and $8.5 \mathrm{mIU} / \mathrm{ml}$ (reference range: $8.3-20.0$ ), respectively, and a low plasma estradiol level $(10.0 \mathrm{pg} / \mathrm{ml}$; reference range, 11.0-172). The secretion of the other pituitary hormones was normal. The chromosome Gbanding analysis showed 46,XX. Intravenous olfactometry (alinamin test) induced no response, indicating anosmia. Brain magnetic resonance imaging (MRI) revealed olfactory bulb agenesis but no abnormalities in the hypothalamus or pituitary (Fig. 1c, d). Her parents and older brother had normal height and age-appropriate pubertal development. Finally, this case was diagnosed as having WS4 and KS.

We received approval for the genetic study from the Ethics Committee of Ehime University Graduate School of Medicine and the Ethics Committee of Keio University School of Medicine. After obtaining written informed consent from her parents, we extracted genomic DNA from peripheral blood samples from the patient. We analyzed major causative genes and possible causative genes for $\mathrm{KS}$ and/or $\mathrm{HH}$, i.e., $A X L, C C D C 141, C H D 7$, DMXL2, DUSP6, FEZF1, FGF8, FGF17, FGFR1, FLRT3, FSHB, GH1, GHR, GHRH, GHRHR, GHSR, GLI2, GNRH1, GNRHR, GPR161, HESX1, HS6ST1, IGF1, IGF1R, IL17RD, KAL1, KISS1, KISS1R, LHB, LHX3, LHX4, LEP, LEPR, MKRN3, NELF, OTUD4, OTX2, PAX6, PCSK1, PGM1,

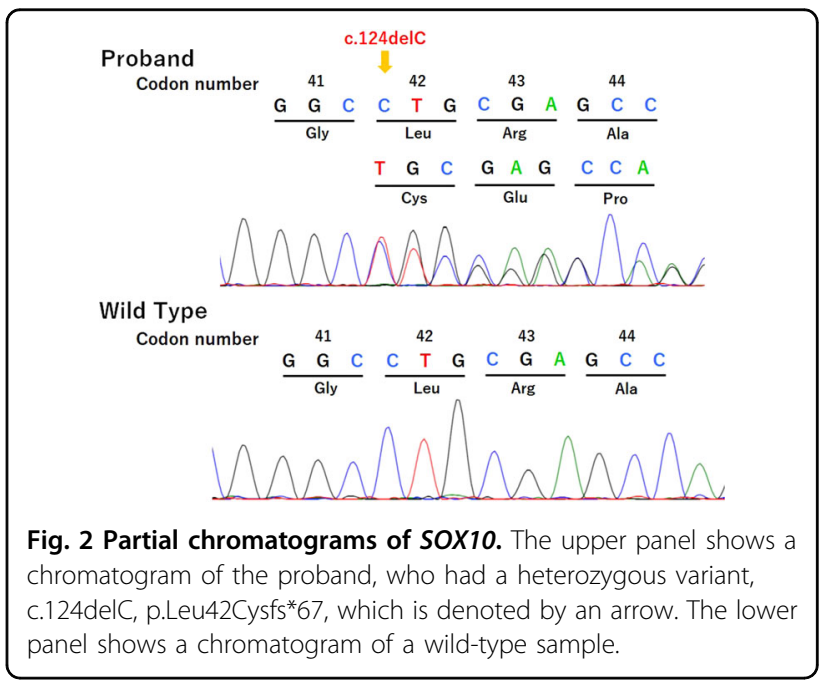

PNPLA6, POLR3A, POLR3B, POU1F1, PROK2, PROKR2, PROP1, RNF216, SEMA3A, SEMA7A, SIX3, SIX6, SOX2, SOX10, SPRY4, STAT5B, STUB1, TAC3, TACR3, TBX19, and TUBB3 using next generation sequencing on a MiSeq instrument (Illumina, San Diego, CA, USA), according to the SureSelect protocol (Agilent Technologies, Santa Clara, CA, USA). We identified a novel heterozygous variant, c.124delC, p.Leu42Cysfs*67, in exon 3 in SOX10 (Fig. 2). This variant was not found in the Human Genetic Variation Database (http://www.hgvd. 
genome.med.kyoto-u.ac.jp/), the gnomAD browser (https://gnomad.broadinstitute.org/), or the 1000 Genome Browser (https://www.ncbi.nlm.nih.gov/variation/tools/ 1000 genomes/). No further pathogenic variant was identified in the other tested genes.

This is the first case of a patient with both WS4C and KS. We, however, cannot deny the possibility that previous reports of WS4C have not been well investigated for gonadal function. When the patient is diagnosed with WS4C in infancy, it is impossible to determine whether the patient has hypogonadism unless he or she is followed until pubertal age.

A novel heterozygous variant, c.124delC, p.Leu42Cysfs"67, in SOX10 was responsible for the WS4C and KS in this case. This frameshift mutation results in a stop codon, theoretically leading to nonsense-mediated messenger RNA decay, and probably causes haploinsufficiency of SOX10. The nonsense mutation p.Glu189* in exon 4 in SOX10 has been reported in a patient with $\mathrm{WS}^{12}$ and in a patient with KS, WS1, and hyperthyroidism ${ }^{13}$. Notably, Inoue et al. ${ }^{14}$ reported that the p.Glu189* and p.Tyr207* mutations in exon 4 in SOX10 showed nonsense-mediated messenger RNA decay. Izumi et al. ${ }^{15}$ determined that the p.Pro169fs"117 mutation in SOX10, which was found in a female patient diagnosed with KS and WS2, was haploinsufficient by an in vitro assay. Amato et al. $^{16}$ reported a large heterozygous deletion involving the entire coding region of SOX10 in a woman with KS and bilateral sensorineural hearing loss. These findings together with the present case suggest that SOX10 haploinsufficiency has wide clinical variation.

In summary, we identified a novel SOX10 variant in a Japanese girl first diagnosed with WS4C and KS. Patients with WS4C due to a SOX10 variant should be examined and followed for clinical features of KS.

\section{HGV Database}

The relevant data from this Data Report are hosted at the Human Genome Variation Database at https://doi.org/10.6084/m9.figshare.hgv.2906.

\section{Acknowledgements}

The authors thank the patient and her family for their participation in this study.

\begin{abstract}
Author details
'Department of Pediatrics, Ehime University Graduate School of Medicine, Ehime, Japan. ${ }^{2}$ Department of Pediatrics, Ehime Prefectural Niihama Hospital, Ehime, Japan. ${ }^{3}$ Department of Pediatrics, Ehime Prefectural Central Hospital, Ehime, Japan. ${ }^{4}$ Department of Pediatrics, Keio University School of Medicine, Tokyo, Japan
\end{abstract}

\section{Author contributions}

J.H., F.O., and Y.S. managed the patient and prepared the manuscript. M.H., H.S., and T.H. performed the molecular analyses. K.T., H.H., T.H., and M.E. supported the manuscript preparation. All authors read and approved the final manuscript.

\section{Conflict of interest}

Tomonobu Hasegawa has the following financial relationships to disclose: Research funding from Novo Nordisk Pharma Ltd. and JCR Pharmaceuticals Co., Ltd.

\section{Publisher's note}

Springer Nature remains neutral with regard to jurisdictional claims in published maps and institutional affiliations.

Received: 21 August 2020 Revised: 1 September 2020 Accepted: 1 September 2020.

Published online: 28 September 2020

\section{References}

1. Pingault, $V$. et al. Review and update of mutations causing Waardenburg syndrome. Hum. Mutat. 31, 391-406 (2010).

2. Chaoui, A. et al. Identification and functional analysis of SOX10 missense mutations in different subtypes of Waardenburg syndrome. Hum. Mutat. 32, 1436-1449 (2011).

3. Hou, L., Arnheiter, H. \& Pavan, W. J. Interspecies difference in the regulation of melanocyte development by SOX10 and MITF. Proc. Natl Acad. Sci. USA 103, 9081-9085 (2006).

4. Barraud, P., St John, J. A., Stolt, C. C., Wegner, M. \& Baker, C. V. Olfactory ensheathing glia are required for embryonic olfactory axon targeting and the migration of gonadotropin-releasing hormone neurons. Biol. Open 2, 750-759 (2013).

5. Kuhlbrodt, K., Herbarth, B., Sock, E., Hermans-Borgmeyer, I. \& Wegner, M. SOX10, a novel transcriptional modulator in glial cells. J. Neurosci. 18, 237-250 (1998).

6. Quinton, R. et al. Idiopathic gonadotrophin deficiency: genetic questions addressed through phenotypic characterization. Clin. Endocrinol. (Oxf.) 55, 163-174 (2001).

7. Marlin, S. et al. Discovery of a large deletion of KAL 1 in 2 deaf brothers. Otol. Neurotol. 34, 1590-1594 (2013).

8. Costa-Barbosa, F. A. et al. Prioritizing genetic testing in patients with Kallmann syndrome using clinical phenotypes. J. Clin. Endocrinol. Metab. 98, E943-E953 (2013).

9. Suzuki, E. et al. De novo frameshift mutation in fibroblast growth factor 8 in a male patient with gonadotropin deficiency. Horm. Res Paediatr. 81, 139-144 (2014).

10. Miraoui, H. et al. Mutations in FGF17, IL17RD, DUSP6, SPRY4, and FLRT3 are identified in individuals with congenital hypogonadotropic hypogonadism. Am. J. Hum. Genet. 92, 725-743 (2013).

11. Pingault, $V$. et al. Loss-of-function mutations in SOX10 cause Kallmann syndrome with deafness. Am. J. Hum. Genet. 92, 707-724 (2013).

12. Pingault, $\mathrm{V}$. et al. SOX10 mutations in patients with WaardenburgHirschsprung disease. Nat. Genet. 18, 171-173 (1998).

13. Wang, F., Zhao, S., Xie, Y., Yang, W. \& Mo, Z. De novo SOX10 nonsense mutation in a patient with Kallmann syndrome, deafness, iris hypopigmentation, and hyperthyroidism. Ann. Clin. Lab Sci. 48, 248-252 (2018).

14. Inoue, K. et al. Molecular mechanism for distinct neurological phenotypes conveyed by allelic truncating mutations. Nat. Genet. 36, 361-369 (2004).

15. Izumi, Y. et al. Hypogonadotropic hypogonadism in a female patient previously diagnosed as having Waardenburg syndrome due to a SOX10 mutation. Endocrine 49, 553-556 (2015).

16. Amato, L. G. L. et al. New genetic findings in a large cohort of congenital hypogonadotropic hypogonadism. Eur. J. Endocrinol. 181, 103-119 (2019). 